\title{
Exploring the impact of adverse childhood experiences on symptomatic and functional outcomes in adulthood: advances, limitations and considerations
}

\author{
J. Cotter ${ }^{1, *}$ and A. R. Yung ${ }^{2,3}$ \\ ${ }^{1}$ Cambridge Cognition, Cambridge, UK \\ ${ }^{2}$ Division of Psychology and Mental Health, Faculty of Biology, Medicine and Health, University of Manchester, Manchester, UK \\ ${ }^{3}$ Greater Manchester Mental Health NHS Foundation Trust, Manchester, UK
}

\begin{abstract}
Exposure to traumatic experiences in childhood is a risk (and potentially causal) factor for the development of a range of adverse physical and mental health conditions. In addition to the onset of clinical disorders, there is emerging evidence that childhood trauma may also be associated with other long-term outcomes, such as the persistence and severity of an individual's symptoms, as well as their long-term social and occupational functioning. However, the reasons for this remain poorly understood. A greater understanding both of the mediators that drive these associations, and those variables that enhance resilience against such damaging experiences may help to inform effective therapeutic interventions. In addition to biological and cognitive measures, there is a need to consider social and environmental factors, such as parental bonding and attachment, when investigating these complex relationships.
\end{abstract}

First published online 18 October 2017

Key words: Attachment, functioning, symptoms, trauma.

Trauma is a broad term in the research literature that encompasses exposure to a range of maladaptive experiences including neglect, and physical, emotional and sexual abuse. There is a growing consensus that exposure to traumatic experiences in childhood is a risk (and potentially causal) factor for the development of a range of adverse physical and mental health outcomes, including psychiatric disorders, obesity, self-injurious behaviour and addiction (Gilbert et al. 2009; Chen et al. 2010; Varese et al. 2012). In addition to the onset of these disorders, there is emerging evidence that childhood trauma may be associated with other long-term clinical outcomes, such as the persistence and severity of an individual's symptoms (Hovens et al. 2012). Recent studies have reported that trauma exposure is also a potential driver of functional problems, not only in childhood, but across the lifespan (Zielinski, 2009). This is evident even among individuals with emerging and full-threshold psychiatric disorders, indicating that exposure to traumatic experiences has enduring detrimental effects that go beyond clinical symptoms, affecting their wider social and occupational functioning (Cotter et al. 2015; Yung et al. 2015). However, the factors that mediate this association remain poorly understood. Greater awareness of these mediators and the variables that enhance resilience against such

* Address for correspondence: J. Cotter, Cambridge Cognition, Tunbridge Court, Bottisham, Cambridge, CB25 9TU, UK.

(Email: jack.cotter@camcog.com) damaging experiences may help to inform effective therapeutic interventions.

In this issue of the Irish Journal of Psychological Medicine, Marshall et al. (2018) report the findings of a study examining the relationships between trauma exposure and parental bonding in childhood with depressive symptoms and interpersonal functioning in adulthood among a sample of patients with bipolar and major depressive disorders. Consistent with previous research, high rates of exposure to traumatic experiences in childhood were reported among both of the diagnostic groups (74\% and $82 \%$, respectively). 'Affectionless control' was the most commonly reported parenting style (compared with 'optimal', 'affectionate constraint' and 'neglectful'). The authors reported that this parental style in conjunction with greater childhood trauma exposure was associated with significantly greater concurrent depressive symptoms and worse social functioning in adulthood among their patient group. However, only the 'affectionless control' variable remained as a significant independent predictor in multivariate analyses.

Though it was not formally assessed in this cohort, parental bonding represents both a potentially important mediating and moderating variable when exploring the relationship between trauma exposure in childhood and long-term clinical and functional outcomes. This is particularly relevant from an attachment perspective, given that exposure to maltreatment at the hands of caregivers is also likely associated with 
insecure attachment between the child and those individuals. Adverse attachment styles are thought to have negative implications for therapy among individuals with psychiatric disorders, impacting on therapeutic alliance, treatment adherence and engagement with clinical services (Gumley et al. 2014; Cotter et al. 2015). This may partially explain the association between trauma history and poorer symptomatic and functional outcomes observed in this and many other cohorts, given that individuals may be less willing to seek help, engage with therapy or take prescribed medication. In contrast, a safe and supportive family environment may help to buffer the impact of other childhood adversities, such as bullying or victimization at the hands of someone outside the family (Jaffee et al. 2017).

There is increasing evidence that routine evaluation of trauma history should be adopted for individuals presenting to mental healthcare services in order to identify those who may require more intensive monitoring and additional treatment. Despite studies suggesting that such assessment and treatment may confer benefits for a range of outcomes (Cotter et al. 2017), routine clinical inquiry about childhood maltreatment is frequently overlooked and inconsistently recorded across mental healthcare settings (Sampson \& Read, 2017). Though clinicians may be reluctant to address this (due, e.g., to fear of symptom exacerbation), there are a number of tools available for the standardized assessment of childhood trauma, ranging from brief self-report screening questionnaires to detailed semistructured interviews (for a review, see Thabrew et al. 2012). Recent research among patients with psychosis reported that trauma-focussed treatment did not result in symptom exacerbation or an increase in adverse events among those individuals randomized to receive the therapy relative to a group of waiting-list controls (van den Berg et al. 2016).

Trauma-focussed clinical interventions are available and have been shown to be effective in reducing posttraumatic stress disorder symptoms among people with serious mental illnesses (Mueser et al. 2015). However, it is also important to consider that childhood trauma may have a number of detrimental effects on individuals, impacting on brain development and cognition, interpersonal behaviour and clinical symptoms, potentially requiring a complex and individually tailored approach to treatment. In their article, Marshall et al. (2017) also cite the potential importance of preventative measures, such as therapeutic intervention aimed at sufferers of past abuse, neglect and poor parenting to prevent 'trans-generational patterns' continuing with their own children.

The study by Marshall et al. (2017) does however suffer from limitations which are typical of much of the existing research in this area. They include a small sample and cross-sectional study design in which patients were asked to retrospectively self-report traumatic childhood experiences and their perception of being parented. The authors briefly refer to the issue of recall bias. It is also worth noting that 'effort after meaning' may have occurred, in which patients may attempt to understand why they became ill and so possibly recall more abuse and harsher parenting than those who are not ill. In addition, many individuals declined to participate and the most symptomatic individuals were excluded from the study. It is unclear whether these various biases would contradict or further support the results of the study. However, it is hard to see how these biases can be avoided in research about maltreatment and parenting, barring a large comprehensive prospective birth cohort study that focusses on these areas.

As Marshall et al. highlight, there is a need to routinely screen and treat those individuals who have experienced trauma, however, there also remain a number of poorly understood factors in the current research literature. For example, while many existing tools (such as the CTQ) provide a useful screening measure to assess the presence of childhood maltreatment, they do not permit detailed evaluation of specific aspects of trauma exposure. Factors such as the age at onset, as well as the frequency and distress associated with exposure to these traumatic experiences are not assessed. The toxic effect of childhood trauma is likely to be exacerbated by sustained and repeated abuse, yet the impact of adulthood revictimization has also been largely overlooked throughout the literature to date (Cotter et al. 2016). These are important considerations that may help to explain some of the individual differences observed in long-term symptomatic and functional outcomes among individuals who have experienced trauma. Evaluation of these issues may permit more detailed conclusions to be drawn on the impact of the timing and specific aspects of trauma exposure on the severity and persistence of adverse clinical outcomes.

In summary, there is a need for studies that seek to address the mechanistic relationship between childhood trauma and both long-term symptomatic and functional outcomes, through comprehensive investigation and sophisticated modelling of factors that potentially drive these associations. In addition to biological and cognitive measures, the paper by Marshall et al. (2017) highlights the need to consider social and environmental factors, such as parental bonding and attachment, when considering these complex relationships given their potential to serve both as important moderators and mediators of these associations. This in turn may have the potential to help inform effective therapeutic interventions. 


\section{Financial Support}

None.

\section{Conflicts of Interest}

None.

\section{Ethical Standards}

The authors assert that all procedures contributing to this work comply with the ethical standards of the relevant national and institutional committee on human experimentation with the Helsinki Declaration of 1975, as revised in 2008.

\section{References}

Chen LP, Murad MH, Paras ML, Colbenson KM, Sattler AL, Goranson EN, Elamin MB, Seime RJ, Shinozaki G, Prokop LJ, Zirakzadeh A (2010). Sexual abuse and lifetime diagnosis of psychiatric disorders: systematic review and meta-analysis. Mayo Clinic Proceedings 85, 618-629.

Cotter J, Drake RJ, Yung AR (2016). Adulthood revictimization: looking beyond childhood trauma. Acta Psychiatrica Scandinavica 134, 368.

Cotter J, Kaess M, Yung AR (2015). Childhood trauma and functional disability in psychosis, bipolar disorder and borderline personality disorder: a review of the literature. Irish Journal of Psychological Medicine 32, 21-30.

Cotter J, Lin A, Drake RJ, Thompson A, Nelson B, McGorry P, Wood SJ, Yung AR (2017). Long-term employment among people at ultra-high risk for psychosis. Schizophrenia Research 184, 26-31.

Gilbert R, Widom CS, Browne K, Fergusson D, Webb E, Janson S (2009). Burden and consequences of child maltreatment in high-income countries. Lancet 373, 68-81.

Gumley AI, Taylor HE, Schwannauer M, MacBeth A (2014). A systematic review of attachment and psychosis: measurement, construct validity and outcomes. Acta Psychiatrica Scandinavica 129, 257-274.

Hovens JG, Giltay EJ, Wiersma JE, Spinhoven P, Penninx BW, Zitman FG (2012). Impact of childhood life events and trauma on the course of depressive and anxiety disorders. Acta Psychiatrica Scandinavica 126, 198-207.

Jaffee SR, Takizawa R, Arseneault L (2017). Buffering effects of safe, supportive, and nurturing relationships among women with childhood histories of maltreatment. Psychological Medicine, doi: 10.1017/S0033291717001027.

Marshall M, Shannon C, Meenagh C, McCorry N, Mulholland C (2018). The association between childhood trauma, parental bonding and depressive symptoms and interpersonal functioning in depression and bipolar disorder. Irish Journal of Psychological Medicine 35, 23-32.

Mueser KT, Gottlieb JD, Xie H, Lu W, Yanos PT, Rosenberg SD, Silverstein SM, Duva SM, Minsky S, Wolfe RS, McHugo GJ (2015). Evaluation of cognitive restructuring for post-traumatic stress disorder in people with severe mental illness. British Journal of Psychiatry 206, 501-508.

Sampson M, Read J (2017). Are mental health staff getting better at asking about abuse and neglect? International Journal of Mental Health Nursing 26, 95-104.

Thabrew H, de Sylva S, Romans SE (2012). Evaluating childhood adversity. Advances in Psychosomatic Medicine 32, 35-57.

van den Berg DP, de Bont PA, van der Vleugel BM, de Roos C, de Jongh A, van Minnen A, van der Gaag M (2016). Trauma-focused treatment in PTSD patients with psychosis: symptom exacerbation, adverse events, and revictimization. Schizophrenia Bulletin 42, 693-702.

Varese F, Smeets F, Drukker M, Lieverse R, Lataster T, Viechtbauer W, Read J, van Os J, Bentall RP (2012). Childhood adversities increase the risk of psychosis: a meta-analysis of patient-control, prospective- and cross-sectional cohort studies. Schizophrenia Bulletin 38, 661-671.

Yung AR, Cotter J, Wood SJ, McGorry P, Thompson AD, Nelson B, Lin A (2015). Childhood maltreatment and transition to psychotic disorder independently predict longterm functioning in young people at ultra-high risk for psychosis. Psychological Medicine 45, 3453-3465.

Zielinski DS (2009). Child maltreatment and adult socioeconomic well-being. Child Abuse \& Neglect 33, 666-678. 